\title{
Do your eyes give it away? Using eye tracking data to understand students' attitudes towards open student model representations
}

\author{
Moffat MATHEWS ${ }^{1}$, Antonija MITROVIC ${ }^{2}$, Bin LIN, Jay HOLLAND and \\ Neville CHURCHER \\ 1 moffat.mathews@canterbury.ac.nz \\ 2 tanja.mitrovic@canterbury.ac.nz \\ Intelligent Computer Tutoring Group, University of Canterbury, Christchurch, \\ New Zealand
}

\begin{abstract}
There is sufficient evidence to show that allowing students to see their own student model is an effective learning and metacognitive strategy. Different tutors have different representations of these open student models, all varying in complexity and detail. EER-Tutor has a number of open student model representations available to the student at any particular time. These include skill meters, kiviat graphs, tag clouds, concept hierarchies, concept lists, and treemaps. Finding out which representation best helps the student at their level of expertise is a difficult task. Do they really understand the representation they are looking at? This paper looks at a novel way of using eye gaze tracking data to see if such data provides us with any clues as to how students use these representations and if they understand them.
\end{abstract}

Keywords: open student modelling, eye tracking, gaze tracking, intelligent tutoring systems, metacognition

\section{Introduction}

A student model is how an Intelligent Tutoring System (ITS) views a student, or more precisely, views their domain knowledge. ITSs use this model to make pedagogical decisions for each student. The student model is not visible to the student. However, it has been shown that opening up the student model to the student, so that they could view "what the system thinks of them" is conducive to learning. In fact, the Open Student Model (OSM) plays quite a large role in increasing their metacognitive skills, which in turn helps their long-term learning $[2,11]$. Opening up the student model means that ITS authors have to consider how to best visualise this data so that the student can understand and make use of it.

As research continues in this area, there are now several new visualisations of the OSM, each giving different details, at different levels, and using different representations. Skill meters $[2,11]$ have been used in a number of systems. 
Other types of OSMs include a tree structure [7,11], and concept graphs [5]. Most of these models are dynamic; others can be interactive; such as the negotiable student model [15]. With so many proposed OSM representations, new questions now exist, such as, "Do students actually understand these representations?", "Can we tell which representations they find easier to understand than others?", or "Are certain representations better for certain populations? e.g. novices versus experts?". If we, as ITS authors, could get the answers to these questions, we could 1) design better, more comprehensible representations, and 2) figure out which representations suit the particular student and guide them towards viewing that one. In this paper, we make an attempt at answering some of these questions for four of the representations contained in an ITS, namely EER-Tutor, in the hope that these methods could then be used to test other OSM representations.

Our method utilises eye (gaze) tracking in combination with test results to see if the student actually understood the model they were presented. Gaze tracking gives us an indication of where the student is looking and what they are paying attention to, while trying to understand the problem and the model. Gaze tracking has been used previously to find comparisons between novices and experts; e.g. during Visual Flight Rules flight [6], during laparoscopic surgery [8], working within a Learning Management System [14], while playing chess [3], and within collaborative environments [9]. It has been found to be a good indicator of the "Yes!" moments of delight while a student interacts with an ITS [13]. Gaze tracking data has also been used to supplement and change the underlying student model $[4,10]$. Bull, Cooke and Mabbott [1] found that students spend more attention on certain OSM representations for a reason, and that developers must take visual gaze attention into account when creating and presenting student models.

In this paper, we want to find out if gaze data gives us any information on how difficult a student finds and understands an OSM representation. For this, we had students viewing four different OSM representations and answering questions on each, while eye gaze data was recorded for each student. We looked at the scores of their answers and compared it to eye gaze data. We believe that if eye gaze data gives us information on how much difficulty a student is having with a particular model, we could, in time, incorporate eye gaze data to dynamically inform the pedagogical module of each student's experience with a certain OSM representation. The score in that future case would be the knowledge score taken from the student's model. The tutor can then intervene and present them with other options of OSM representations.

\section{Design and Methodology}

Seventeen participants took part in this study. They were all students who belonged to a second-year database course at the University of Canterbury. Each participant was given a NZ $\$ 20$ voucher on completion of the study. The ITS chosen for this study was EER-Tutor [12] and the study was conducted using 
a Tobii TX300 $3(300 \mathrm{~Hz})$ eyetracker. Each participant took part in the study separately.

Fig. 1. Gazeplot for the kiviat and concept tag graphs

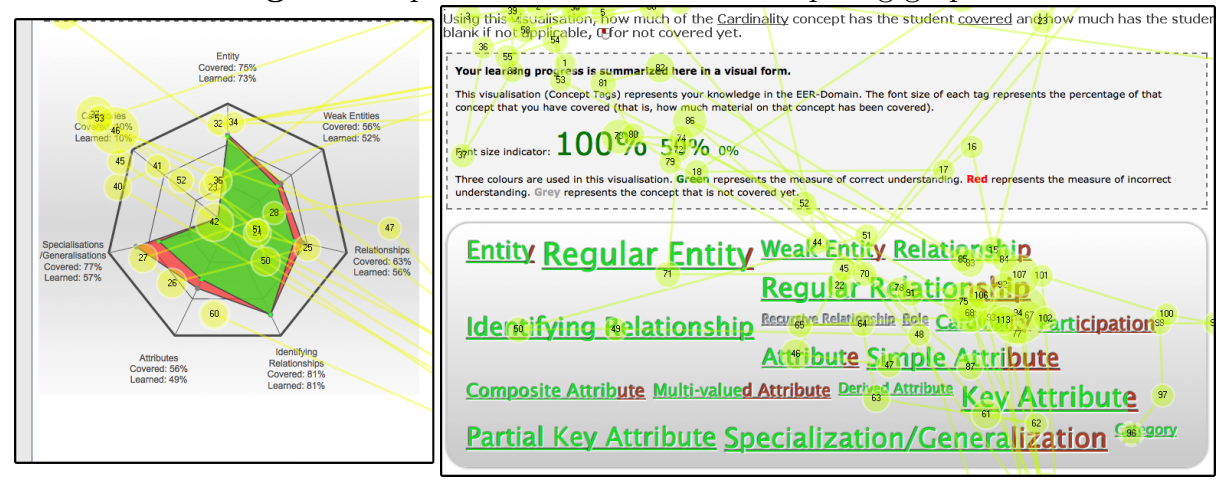

EER-Tutor is a standard tutor that students use in the lab sessions of this course; the lab sessions occur after the relevant lectures on the topic at hand. All participants had logged into EER-Tutor once during the first EER lab and completed the pretest. The version used in the course was similar in all respects to that used in the study, except that it only had one OSM representation: the skill meter.

Each participant took approximately an hour to complete the study. After the initial formalities of the study (information, consent form, etc.), each participant was asked to spend twenty minutes on the evaluation version of EER-Tutor. This version had all four OSM representations: kiviat chart; concept tags; concept hierarchy; and treemap. Participants were instructed to try solving problems, but to mainly focus on understanding each of the representations. During these twenty minutes, there were no restrictions put on the participants; they could solve as many or as few problems as they wished, as long as they focused on understanding the OSM representations.

After the twenty minute session, the participants were automatically redirected to a web survey that we created, where they were asked questions about each of the OSM representations. To keep the eye gaze data clean and separated, all questions relating to a particular OSM representation were on the same page; the eyetracker generated new eye gaze plots for each page. Each page had a different representation of a pre-made model; all participants received the same pre-made OSMs. There were three questions directly related to each OSM and the understanding of the OSM in terms of the domain. As an example of a question, participants were asked to view an OSM and answer how much the student

\footnotetext{
${ }^{3}$ http://www.tobii.com/en/eye-tracking-research/global/products/hardware/tobiitx300-eye-tracker
} 
Fig. 2. Gazeplot for the treemap and the concept hierarchy
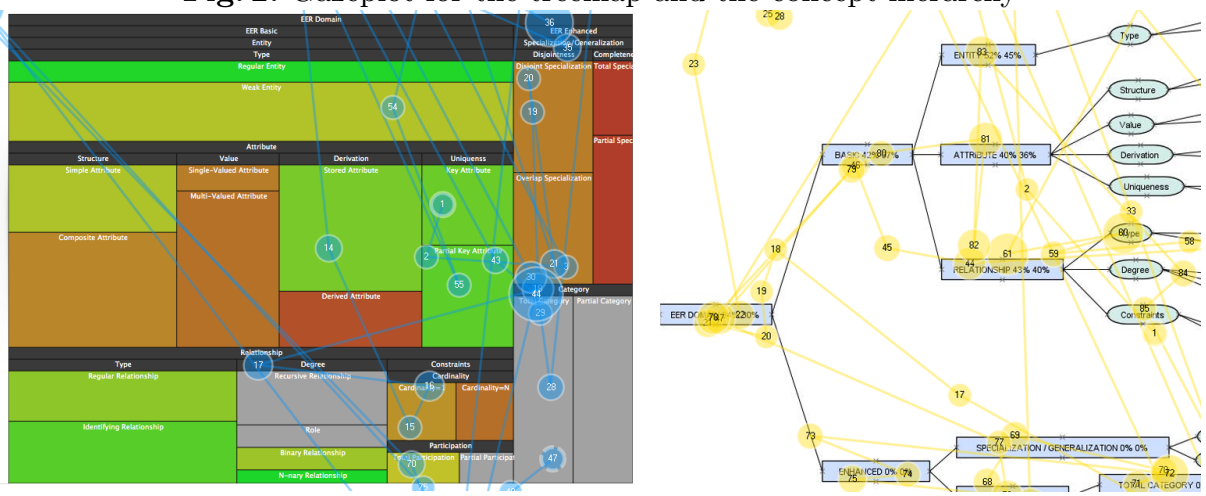

(represented by the OSM) had learnt and covered for a particular concept. Each question then could be given a score and participants were marked accordingly. At the end of the questionnaire there were two unmarked questions where participants could: 1) give general feedback and 2) rank their preference of the OSMs in the context of learning. Once a participant moved on from a page, they could not go back and change their answers. Participants were allowed to see their gaze data after completion of the evaluation study.

We have included cropped figures to show examples of the gazeplots from one question for one participant for each of the OSMs; Fig. 1 for the kiviat graph and the tag clouds, Fig. 2 for the concept hierarchy, and the treemap. Each node in the gaze data is a fixation. The longer the fixation, the bigger the node.

Our idea for this research was to find if eye gaze data added any value to figuring out how quickly and efficiently a student understood a particular OSM. If eye gaze data could be used in such a manner, then ITSs in future could track a student using one version of the OSM, figure out if they are having difficulty with it, and then intervene in some way, such as presenting them with a different OSM.

\section{Results and Discussion}

We defined a new variable called OSM Efficiency. The more efficient someone was at understanding a particular model, the higher their score would be. They would also be able to understand the OSM in less time with fewer fixations. With this logic, we came up with our equation for OSM Efficiency, which is given in Equation 1.

$$
\text { OSM Efficiency }=\frac{\text { Score }}{\text { Time } \times \text { Number of fixations }}
$$

An expert marked the answers to the OSM questions according to the marking schema and came up with a score for each OSM. The time and number of fixations were extracted from the eyetracker. 
We used repeated measures ANOVA and found a significant differences between the efficiencies in the OSM groups $(F(3,42)=43.567, p>.05)$. To find out which groups were significantly different from each other, we conducted a Bonferroni post-hoc test. There is a significant difference between the kiviat graph and two other OSMs (tag cloud and treemap). There is no difference between kiviat graph and concept hierarchy. Similarly, there is a significant difference between concept hierarchy and two other OSMs (tag cloud and treemap). There is no difference between tag cloud and treemap.

This shows that participants were on average more efficient (with our definition of efficiency) using the kiviat graph and the concept hierarchy, but had difficulties understanding and answering questions using the tag cloud and treemap representations.

However, how does this match up with participants' attitudes towards the OSM representations? In our questionnaire, we asked participants to rank the OSMs according to their preference in a learning context.

There was a statistically significant difference in the rankings of the OSMs $(\chi 2(3)=17.118, p=0.001)$. Post-hoc analysis with Wilcoxon Signed-Rank Tests was conducted with a Bonferroni correction applied, resulting in a significance level set at $p<0.0125$. Median (IQR) ranking levels were 1.0 (1.0 to 2.5) for kiviat graph, 3.0 (2.0 to 4.0) for tag clouds, 2.0 (2.0 to 3.0) for concept hierarchy, and 4.0 (3.0 to 4.0$)$ for treemap. There was a statistically significant difference in tag cloud vs. kiviat graph rankings $(Z=-2.545, p=.011)$, and in treemap vs. kiviat graph rankings $(Z=-3.103, p=0.002)$.

The comments' question gave participants a chance to tell us about their experience with the OSMs. Many agreed that the Kiviat Chart was best for an overall and quick indication of their levels but the other representations had their uses if more information was required. This led to the conclusion that the best OSMs depend on the context of the situation.

ITS designers are becoming more creative with their OSM designs. There has to be a method of testing between the various OSMs rather than just assuming that all OSMs are easy to understand. In this paper, we were able to compare four OSMs and found significant differences between them in terms of efficiency. This efficiency took into account the participant's score, their time for fixations, and their number of fixations. Future ITSs would gather the student's knowledge score (from the student model) instead of a questionnaire score to determine if the student is having difficulties. We compared this with subjective questionnaires that the participants had submitted rating their preference for each of the OSMs. There were significant commonalities between the efficiencies and the preferences. Furthermore, we manually analysed their comments and found that their attitude towards the OSMs were significantly similar to both the efficiencies and preferences. Following on from the background research and the participants' comments, we wonder if there would be a difference between different groups of students (say, novices versus experts). Novices might be interested in an easy to understand smaller OSM, while experts might want further detail and not be content with the smaller OSMs. We also found that eye tracking can 
play a large role in automatically understanding how the student is feeling towards each OSM. This could later on be harnessed with ITSs to present students with different OSMs when the ITS notices that they are struggling using their eye gaze data.

\section{References}

1. Bull, S., Cooke, N., Mabbott, A.: Visual attention in open learner model presentations: An eye-tracking investigation. In: Conati, C., McCoy, K., Paliouras, G. (eds.) User Modeling 2007, pp. 177-186. Springer Berlin / Heidelberg (2007)

2. Bull, S., Quigley, S., Mabbott, A.: Computer-based formative assessment to promote reflection and learner autonomy. Engineering Education: Journal of the Higher Education Academy Engineering Subject Centre 1(1), 8-18 (2006)

3. Charness, N., Reingold, E.M., Pomplun, M., Stampe, D.M.: The perceptual aspect of skilled performance in chess: evidence from eye movements. Memory \& Cognition 29(8), 1146-1152 (2001)

4. Conati, C., Merten, C., Muldner, K., Ternes, D.: Exploring eye tracking to increase bandwidth in user modeling. In: Ardissono, L., Brna, P., Mitrovic, A. (eds.) User Modeling 2005, vol. 3538, pp. 151-151 (2005)

5. Dimitrova, V.: Style-olm. Artificial Intelligence in Education 13(2), 35-78 (2003)

6. Kasarskis, P., Stehwien, J., Hickox, J., Aretz, A., Wickens: Comparison of expert and novice scan behaviors during vfr flight. In: 11th Int. Symposium on Aviation Psychology (2001)

7. Kay, J.: Learner know thyself: Student models to give learner control and responsibility. In: Proc. of the Int. Conf. on Computers in Education. pp. 17-24. AACE, Charlottesville, VA (1997)

8. Law, B., Atkins, M.S., Kirkpatrick, A.E., Lomax, A.J.: Eye gaze patterns differentiate novice and experts in a virtual laparoscopic surgery training environment. In: Proc. 2004 symposium on Eye tracking research \& applications. pp. 41-48. ETRA '04, ACM, New York, NY, USA (2004)

9. Liu, Y., Hsueh, P.Y., Lai, J., Sangin, M., Nussli, M.A., Dillenbourg, P.: Who is the expert? analyzing gaze data to predict expertise level in collaborative applications. In: IEEE Int. Conf. on Multimedia and Expo. pp. 898-901 (2009)

10. Merten, C., Conati, C.: Eye-tracking to model and adapt to user meta-cognition in intelligent learning environments. In: Proc. 11th int. conf. Intelligent user interfaces. pp. 39-46. IUI '06, ACM, New York, NY, USA (2006)

11. Mitrovic, A., Martin, B.: Evaluating the effect of open student models on selfassessment. Artificial Intelligence in Education 17(2), 121-144 (2007)

12. Mitrovic, A.: Fifteen years of constraint-based tutors: what we have achieved and where we are going. User Modeling and User-Adapted Interaction pp. 1-34 (2011)

13. Muldner, K., Burleson, W., VanLehn, K.: "yes!": Using tutor and sensor data to predict moments of delight during instructional activities. In: De Bra, P., Kobsa, A., Chin, D. (eds.) UMAP 2010, pp. 159-170 (2010)

14. Pretorius, M., van Biljon, J.: Learning management systems: Ict skills, usability and learnability. Interactive Technology and Smart Education 7(1), 30 - 43 (2010)

15. Thomson, D., Mitrovic, A.: Preliminary evaluation of a negotiable student model in a constraint-based its. Research and Practice in Technology Enhanced Learning (RPTEL) 5(1), 19-33 (2010) 